\title{
Análise do custo do tratamento da ambliopia para o paciente em hospital universitário
}

\author{
A cost analysis of therapy for amblyopia for an outpatient at a university hospital
}

\author{
Luciana Ottaiano Cerântola de Almeida ${ }^{1}$
}

Trabalho realizado na Universidade Federal de São Paulo/Escola Paulista de Medicina - UNIFESP/EPM.

${ }^{1}$ Oftalmologista pela Faculdade de Medicina de Marília. Marília (SP)

Endereço para correspondência: Luciana Ottaiano Cerântola de Almeida. Av. Santo Antônio, 271 - Apto. 1700 - Marília (SP) CEP 17501-470

E-mail: lcerantola@uol.com.br

Recebido para publicação em 16.06.2004

Versão revisada recebida em 03.01.2005

Aprovação em 07.04.2005

\begin{tabular}{l} 
RESUMO \\
\hline Objetivo: Avaliar os custos do tratamento da ambliopia para o paciente \\
pela oclusão do olho dominante, assim como seu resultado visual. \\
Métodos: Realizou-se estudo prospectivo para avaliar o custo do trata- \\
mento de ambliopia por oclusão no Setor de Estrabismo do Hospital São \\
Paulo - Universidade Federal de São Paulo/Escola Paulista de Medicina. \\
Crianças portadoras de ambliopia entre 3 e 7 anos de idade foram \\
incluídas no estudo. Os pacientes foram acompanhados a partir do \\
diagnóstico até três retornos consecutivos. Ao diagnóstico prescreve- \\
ram-se óculos se necessário e oclusão total, direta e contínua do olho \\
dominante. Nos retornos coletaram-se dados referentes ao tratamento e \\
ao custo do tratamento da ambliopia. Para análise dos resultados os \\
custos foram divididos em diretos (óculos e oclusor) e indiretos (trans- \\
porte e desconto do dia de trabalho do acompanhante). Resultados: \\
Foram analisados 14 pacientes com idade média de 5,21 anos, sendo 7 \\
(50\%) do sexo masculino e 7 (50\%) do sexo feminino. Observou-se \\
melhora da acuidade visual em $71,43 \%$ dos pacientes. O custo médio \\
mensal do tratamento da ambliopia foi de R\$ 59,49 e o custo médio anual \\
de R\$714,47. O custo médio mensal do tratamento da ambliopia equivale \\
a $12,20 \%$ da renda familiar mensal média dos pacientes neste estudo. \\
Conclusão: O tratamento de oclusão da ambliopia é eficiente e melhorou \\
a acuidade visual do olho amblíope em $71,43 \%$ dos casos. O custo mensal \\
deste tratamento é de R\$ 59,49 e representa $12,20 \%$ da renda familiar \\
mensal dos pacientes avaliados.
\end{tabular}

Descritores: Ambliopia/terapia; Ambliopia/economia; Acuidade visual/fisiologia; Relação custo-benefício; Privação sensorial; Custos de cuidados de saúde

\section{INTRODUÇ̃̃̃O}

A palavra ambliopia vem do grego e literalmente significa "visão embaçada"(1-2).

A ambliopia é a falha na consolidação da acuidade visual (AV) unilateral ou bilateral decorrente da falta de estímulos ou presença de estímulos inadequados ou insuficientes durante o período crítico do desenvolvimento da visão(3).

A prevalência da ambliopia em crianças é estimada de $1 \%$ a $4 \%$ e é a causa mais comum de redução da acuidade visual na infância ${ }^{(4)}$.

A ambliopia é classificada nos seguintes tipos: ambliopia estrábica, ambliopia refrativa (anisometrópica, ametropias bilaterais, meridional), ambliopia por privação, ambliopia orgânica, ambliopia idiopática e ambliopia associada ao nistagmo ${ }^{(2-3)}$. 
Introduzida há mais de 250 anos, a oclusão permanece até hoje como o principal tratamento para a ambliopia com índices de sucesso variando de $30 \%$ até $93 \%{ }^{(5)}$.

Há uma divergência de opiniões sobre o número de horas de oclusão por dia a ser prescrito, variando de poucas horas até todas as horas de vigília ${ }^{(6)}$.

Para aumentar a melhora da acuidade visual com a terapia de oclusão, a maioria dos especialistas recomendam manter um programa de oclusão com grande número de horas diárias de oclusão durante pelo menos 3 retornos consecutivos. A partir daí, não se obtendo melhora da acuidade visual, pode-se reduzir ou suspender a terapia de oclusão. Tradicionalmente, a duração dos intervalos de oclusão é de uma semana a cada ano de idade da criança ${ }^{(7)}$.

É reconhecido que a terapia de oclusão pode levar o paciente a atingir acuidade visual normal ou quase normal no olho amblíope na maioria dos casos, principalmente quando o tratamento é instituído na infância precoce (até aos 7 anos ou idade escolar) ${ }^{(8)}$.

Apesar da terapia de oclusão ter sucesso em muitos casos, alguns pacientes não obtêm melhora da acuidade visual. As causas para o mau resultado visual incluem: ambliopia irreversível, fraca aderência ao tratamento, anormalidade ocular orgânica associada e esquema de oclusão insuficiente ${ }^{(7)}$.

A terapia de oclusão custa aproximadamente U\$ 100 dólares para 6 meses de tratamento ${ }^{(8)}$.

A eliminação ou redução da ambliopia resulta em benefícios diretos e indiretos para a sociedade. Estudos revelam que a detecção precoce da ambliopia produz economia de U\$ 60.000,00 para cada 1.000 crianças. A economia indireta de custos refere-se à menor quantidade de crianças requerendo programas especiais e cuidados especiais na escola. Ao longo da vida, indivíduos com ambliopia terão limitação em exercer certas profissões e de acordo com a literatura terão maior chance de sofrer injúria no olho sadio ${ }^{(9)}$.

Quando comparado com outros tratamentos em saúde, o tratamento da ambliopia parece ser altamente custo-efetivo. Alguns procedimentos em oftalmologia como fotocoagulação para retinopatia da prematuridade e para neovascularização de coróide têm se mostrado bastante custo-efetivos e outros como paracentese de câmara anterior e inalação de dióxido de carbono para o tratamento de obstrução da artéria central da retina não têm se mostrado especialmente custo-efetivos ${ }^{(10)}$.

O tratamento da ambliopia é uma intervenção realizada no exercício da oftalmologia pediátrica que usualmente produz resultados para o resto da vida dos pacientes ${ }^{(11)}$.

\section{OBJETIVOS}

Avaliar a melhora da acuidade visual e o custo do tratamento da ambliopia para o paciente através da oclusão do olho dominante e relacionar o custo mensal do tratamento da ambliopia com a renda familiar dos pacientes avaliados.

\section{MÉTODOS}

Foi realizado estudo prospectivo para avaliar o custo do tratamento de oclusão da ambliopia no Setor de Estrabismo do Hospital São Paulo - Universidade Federal de São Paulo/Escola Paulista de Medicina no período de 31/07/2002 até 31/09/2003.

Definiu-se ambliopia como diferença de acuidade visual entre o olho dominante e o olho amblíope maior ou igual a duas linhas de visão da tabela de Snellen ${ }^{(12)}$.

Os critérios de inclusão foram: crianças portadoras de ambliopia entre 3 anos e 7 anos de idade, ausência de tratamento prévio para ambliopia, ausência de doenças oculares ou da via visual que pudessem justificar a baixa de acuidade visual e crianças que colaboraram para obtenção da medida de acuidade visual.

Foi realizado exame oftalmológico na primeira consulta, que incluiu: acuidade visual sem e/ou com correção, exame da motilidade ocular extrínseca, biomicroscopia, retinoscopia sob cicloplegia e fundoscopia.

A acuidade visual dos pacientes foi obtida através de optotipos iletrados "E" da tabela de Snellen com notação decimal. Nos casos em que o paciente conseguiu visibilizar uma ou mais letras, mas não todas, de uma das linhas de acuidade visual da tabela de Snellen, foi registrado a acuidade visual obtida seguida da letra "p“ (parcial), Ex.: 0,2p.

A retinoscopia foi realizada trinta a quarenta minutos após a instilação de uma gota de colírio anestésico, seguido de uma gota de colírio de tropicamida e uma gota de colírio de ciclopentolato.

Para cada paciente classificou-se a ambliopia em: estrábica, anisometrópica ou por privação.

Após o exame inicial, prescreveu-se o tratamento: óculos se necessário e oclusão do olho não amblíope de forma total (durante todo período de vigília), direta (sobre a pele) e contínua (todos os dias).

Foi orientado ao acompanhante das crianças os tipos de oclusores que a criança poderia utilizar: caseiro, adesivo ou plástico.

O primeiro retorno foi realizado após o número de semanas equivalente à idade do paciente (Ex.: criança de 3 anos: retorno em 3 semanas) e os retornos subseqüentes a cada 4 semanas aproximadamente, considerando-se as particularidades de cada caso. As crianças que obtivessem a acuidade visual do olho amblíope igual a do olho dominante passavam para oclusão anti-supressiva parcial de 4 a 6 horas por dia.

Os pacientes foram acompanhados a partir do diagnóstico na consulta inicial até 3 retornos subseqüentes.

Nos retornos foi verificado: acuidade visual com e/ou sem correção, exame da motilidade ocular extrínseca, retinoscopia sob cicloplegia se julgado necessário pelo pesquisador.

A obtenção de dados foi realizada pelo próprio pesquisador diretamente com os acompanhantes das crianças.

Os seguintes dados foram coletados na primeira entrevista (consulta inicial): nome, idade, sexo e data de nascimento. 
Nas entrevistas subseqüentes (retornos): custo dos óculos, tipo de oclusor utilizado (adesivo, plástico, caseiro), esquema de oclusão, número de oclusores utilizados por dia, custo do oclusor, identificação do acompanhante da criança, custo do dia de trabalho do acompanhante, meio de transporte utilizado até o setor, custo do transporte, renda familiar mensal, adesão ao tratamento (total, parcial ou nula).

O exame oftalmológico da criança na consulta inicial e nos retornos subseqüentes, assim como as entrevistas com os acompanhantes das crianças foram realizados completamente pelo próprio pesquisador.

Para análise dos resultados do custo do tratamento da ambliopia neste estudo, dividiu-se os custos em diretos e indiretos. Os custos diretos referem-se ao tratamento em si: óculos e oclusor. Os custos indiretos referem-se aos gastos dispendidos para o comparecimento aos retornos: transporte, desconto do dia de trabalho do acompanhante.

Para o cálculo do custo do oclusor adesivo foi considerado o preço da unidade de oclusor e multiplicado este valor pelo número de oclusores utilizados por dia e pelo número de dias de tratamento.

Para o cálculo do custo mensal e anual do tratamento da ambliopia foi considerado o custo diário e multiplicado este valor por 30,41 (mensal) e por 365 (anual), respectivamente.

Este trabalho foi analisado e aprovado pelo Comitê de Ética em Pesquisa da Universidade Federal de São Paulo/ Hospital São Paulo, CEP No.0808/03 (Anexo 2).

\section{RESULTADOS}

Foram analisados 14 pacientes sendo $7(50 \%)$ do sexo feminino e 7 (50\%) do sexo masculino. A idade dos pacientes no início do tratamento variou de 3 anos a 7 anos, com média de 5,21 anos.

O tipo de ambliopia mais freqüente foi a estrábica em 12 $(85,71 \%)$ casos, seguido da anisometrópica em 2 (14,29\%) casos. Nenhum paciente apresentava ambliopia por privação.

A acuidade visual inicial do olho amblíope variou de 0,08 até $0,7 \mathrm{p}$ e a acuidade visual no terceiro retorno variou de 0,08 até 1,0 .

Em $10(71,43 \%)$ casos houve melhora da acuidade visual do olho amblíope e em $4(28,57 \%)$ casos os pacientes permaneceram com a acuidade visual igual a da primeira consulta.

Dos 14 pacientes avaliados, $12(85,71 \%)$ receberam prescrição de óculos na ocasião da primeira consulta, $1(7,14 \%)$ no segundo retorno e $1(7,14 \%)$ não necessitou de óculos durante o tratamento.

Quanto ao tipo de oclusor, $13(92,86 \%)$ pacientes utilizaram o oclusor adesivo e $1(7,14 \%)$ paciente utilizou o oclusor plástico. Dos 13 pacientes que utilizaram o oclusor adesivo, 5 $(38,46 \%)$ utilizaram 1 unidade de oclusor por dia, $6(46,15 \%)$ utilizaram 2 unidades de oclusor por dia, 1 (7,69\%) utilizou 2 ou 3 oclusores por dia e $1(7,69 \%)$ utilizou 3 oclusores por dia.
De acordo com a informação do acompanhante da criança, $8(57,14 \%)$ pacientes realizaram o tratamento de oclusão corretamente (obedecendo ao número de horas de oclusão prescrita) e $6(42,86 \%)$ pacientes realizaram menor tempo de oclusão em relação ao número de horas prescrito.

O transporte utilizado para acesso ao ambulatório de estrabismo no Hospital São Paulo durante o tratamento da ambliopia foi o ônibus em $9(64,28 \%)$ casos e ônibus + metrô em 5 $(35,71 \%)$ casos.

Em relação ao acompanhante da criança, em $10(71,43 \%)$ casos era a mãe, em $1(7,14 \%)$ caso o pai, em $1(7,14 \%)$ caso a irmã, em $1(7,14 \%)$ caso a avó e em $1(7,14 \%)$ caso a madrinha.

Dos 14 acompanhantes das crianças, $9(64,29 \%)$ não possuíam trabalho remunerado e $5(35,71 \%)$ possuíam. Em nenhum caso foi descontado dia de trabalho do acompanhante. Quando solicitado, forneceu-se atestado comprovando o comparecimento dos mesmos nas consultas médicas.

A renda familiar das 14 crianças variou de $\mathrm{R} \$ 250,00$ até $\mathrm{R} \$ 800,00$ com média de $\mathrm{R} \$ 560,57$, e a renda "per capita" variou de $R$ \$ 53,60 até $R$ \$243,33 com média de $R$ \$ 144,61.

O tempo de acompanhamento das crianças variou de 12 semanas a 15 semanas com média de 13,4 semanas.

No presente estudo, o custo do tratamento da ambliopia para o paciente a partir do seu diagnóstico até o terceiro retorno variou de $\mathrm{R} \$ 83,46$ a $\mathrm{R} \$ 268,00$, com média de $\mathrm{R} \$ 181,78$ (Tabela 1).

O custo médio diário do tratamento da ambliopia para o paciente neste estudo foi de $\mathrm{R} \$ 1,95$, o custo médio mensal foi de $\mathrm{R} \$ 59,49$ e o custo médio anual foi de $\mathrm{R} \$ 714,47$ (Tabela 2).

$O$ custo mensal do tratamento da ambliopia neste estudo em relação à renda familiar mensal dos pacientes avaliados variou de $3,95 \%$ a $22,88 \%$ e em relação à renda "per capita" mensal variou de $18,37 \%$ a $98,99 \%$ (Tabela 3 ).

\begin{tabular}{|c|c|c|c|c|}
\hline Paciente & Oclusor & Óculos & Transporte & Total \\
\hline 1 & 52,06 & 165,00 & 7,30 & 224,36 \\
\hline 2 & 43,18 & 100,00 & 15,90 & 159,08 \\
\hline 3 & 35,00 & 170,00 & 9,00 & 214,00 \\
\hline 4 & 45,06 & 76,00 & 9,60 & 130,66 \\
\hline 5 & 63,70 & 110,00 & Carteirinha & 173,70 \\
\hline 6 & 67,06 & Não utiliza & 16,40 & 83,46 \\
\hline 7 & 163,80 & 70,00 & 20,40 & 254,20 \\
\hline 8 & Ganhou & 140,00 & 6,20 & 146,20 \\
\hline 9 & 75,60 & 60,00 & 20,40 & 156,00 \\
\hline 10 & 28,00 & 240,00 & Carteirinha & 268,00 \\
\hline 11 & 122,50 & 85,00 & 10,20 & 217,70 \\
\hline 12 & 44,59 & 89,00 & 20,40 & 153,99 \\
\hline 13 & 46,20 & 154,00 & 25,20 & 225,40 \\
\hline 14 & 48,02 & 80,00 & 10,20 & 138,22 \\
\hline Média * & 64,21 & 118,38 & 14,26 & 181,78 \\
\hline
\end{tabular}




\begin{tabular}{|cccc|}
\hline \multicolumn{4}{|c|}{$\begin{array}{c}\text { Tabela 2. Custos do tratamento da ambliopia para o paciente em } \\
\text { reais: diário, mensal e anual }\end{array}$} \\
Paciente & Custo diário & Custo mensal & Custo anual \\
1 & 2,46 & 74,80 & 897,90 \\
2 & 1,51 & 45,91 & 551,15 \\
3 & 2,86 & 86,97 & $1.043,90$ \\
4 & 1,33 & 40,44 & 490,77 \\
5 & 1,77 & 53,82 & 646,05 \\
6 & 0,91 & 27,67 & 332,15 \\
7 & 2,79 & 84,84 & $1.018,35$ \\
8 & 1,74 & 52,91 & 635,10 \\
9 & 1,85 & 56,25 & 675,25 \\
10 & 2,55 & 77,54 & 930,75 \\
11 & 2,22 & 67,51 & 810,30 \\
12 & 1,69 & 51,39 & 616,85 \\
13 & 2,30 & 69,94 & 839,50 \\
14 & 1,41 & 42,87 & 514,65 \\
Média & 1,95 & 59,49 & 714,47 \\
\hline
\end{tabular}

\section{DISCUSSÃO}

Foi observado melhora da acuidade visual do olho amblíope em $71,43 \%$ dos pacientes analisados. O tempo médio de acompanhamento de cada paciente foi 13,42 semanas ( 3 meses). Outros estudos relataram maiores porcentagens de casos com melhora da acuidade visual do olho amblíope. Alguns autores encontraram melhora de acuidade visual em $99 \%$ dos casos em um estudo de ambliopia que teve um tempo médio de acompanhamento de 30 meses e 16 meses para pacientes com até 6 anos de idade e com mais de 6 anos de idade, respectivamente $^{(12)}$. Um outro encontrou melhora da acuidade visual em $100 \%$ dos pacientes tratados por ambliopia estrábica em um tempo médio de 4,4 meses $^{(13)}$. Um trabalho sobre ambliopia anisometrópica mostrou melhora da acuidade visual em $86,1 \%$ dos casos em um tempo médio de 11,5 meses ${ }^{(14)}$.

É importante considerar que o tempo de acompanhamento dos pacientes neste estudo foi inferior aos estudos citados, o que pode justificar a porcentagem inferior de casos com melhora da acuidade visual.

Todas as crianças deste estudo continuaram em acompanhamento e tratamento da ambliopia, pois este tratamento é geralmente prolongado podendo se estender por meses ou anos. Se este estudo se estendesse por mais tempo, provavelmente teria maior porcentagem de melhora visual que a encontrada $(71,43 \%)$. O período médio de 3 meses de acompanhamento deveu-se ao fato de este estudo ter como principal objetivo a análise do custo do tratamento da ambliopia.

A ambliopia deve ser tratada até que a visão do olho amblíope se iguale à do olho dominante ou até o mínimo de 3 meses de tratamento constante sem melhora da acuidade visual do olho amblíope ${ }^{(12)}$. Foi demonstrado que o tempo necessário para atingir a melhor acuidade visual pode variar de 3 a 46 meses $^{(15)}$. Um estudo encontrou períodos variáveis para atingir a melhor acuidade visual e, em seis pacientes, o tempo encontrado para a resposta máxima ao tratamento variou de 5 meses a 5 anos e 6 meses $^{(16)}$.

O custo do tratamento da ambliopia para o paciente neste estudo compreendeu os custos diretamente relacionados ao tratamento (custo dos óculos e do oclusor) e os custos indiretamente relacionados ao tratamento (custo do transporte até o local do atendimento e do desconto salarial do acompanhante da criança).

No presente estudo acompanhou-se os pacientes a partir do diagnóstico até 3 retornos consecutivos. Obteve-se um custo médio mensal de $\mathrm{R} \$ 59,49$. O custo dos óculos representou em média $60,14 \%$ do custo do tratamento, o custo do oclusor $32,62 \%$ e o custo do transporte $7,24 \%$.

\begin{tabular}{|c|c|c|c|c|c|}
\hline Paciente & $\begin{array}{l}\text { RF mensal } \\
\text { em reais }\end{array}$ & $\begin{array}{l}\text { Custo mensal } \\
\text { do tratamento } \\
\text { da A. em reais }\end{array}$ & $\begin{array}{c}\text { Porcentagem da RF } \\
\text { mensal equivalente ao } \\
\text { custo do tratamento da } A \text {. }\end{array}$ & $\begin{array}{l}\text { RC mensal } \\
\text { em reais }\end{array}$ & $\begin{array}{l}\text { Porcentagem da RC mensal } \\
\text { equivalente ao custo mensal } \\
\text { do tratamento da } A \text {. }\end{array}$ \\
\hline 1 & 600,00 & 74,80 & $12,46 \%$ & 200,00 & $37,40 \%$ \\
\hline 2 & 250,00 & 45,91 & $18,36 \%$ & 83,33 & $55,09 \%$ \\
\hline 3 & 380,00 & 86,97 & $22,88 \%$ & 95,00 & $91,54 \%$ \\
\hline 4 & 750,00 & 40,44 & $5,39 \%$ & 187,50 & $21,56 \%$ \\
\hline 5 & 500,00 & 53,82 & $10,76 \%$ & 166,66 & $32,29 \%$ \\
\hline 6 & 700,00 & 27,67 & $3,95 \%$ & 140,00 & $19,76 \%$ \\
\hline 7 & 780,00 & 84,84 & $10,87 \%$ & 156,00 & $54,38 \%$ \\
\hline 8 & 268,00 & 52,91 & $19,74 \%$ & 53,60 & $98,71 \%$ \\
\hline 9 & 350,00 & 56,25 & $16,07 \%$ & 87,50 & $64,28 \%$ \\
\hline 10 & 470,00 & 77,54 & $16,49 \%$ & 78,33 & $98,99 \%$ \\
\hline 11 & 570,00 & 67,51 & $11,84 \%$ & 142,50 & $47,37 \%$ \\
\hline 12 & 800,00 & 51,39 & $6,42 \%$ & 160,00 & $32,11 \%$ \\
\hline 13 & 730,00 & 69,94 & $9,58 \%$ & 243,33 & $28,74 \%$ \\
\hline 14 & 700,00 & 42,87 & $6,12 \%$ & 233,33 & $18,37 \%$ \\
\hline Média & 560,57 & 59,49 & $12,20 \%$ & 144,79 & $50,04 \%$ \\
\hline
\end{tabular}


Notou-se que o custo do tratamento da ambliopia é cumulativo ao longo do tratamento e isto se deve principalmente ao fato dos oclusores adesivos serem descartáveis e necessitarem de troca diária, muitas vezes mais de uma vez ao dia. $\mathrm{O}$ oclusor plástico é também eficiente e não é descartável, portanto, seu uso pode reduzir o custo do tratamento.

A grande maioria dos pacientes neste estudo optou pela utilização do oclusor adesivo. Provavelmente isto ocorre porque o oclusor adesivo tem um aspecto estético melhor e maior facilidade de ser encontrado (farmácias). Já o oclusor plástico necessita ser comprado em ópticas e às vezes não é fácil de ser encontrado. Além disto, uma unidade do oclusor plástico é mais cara que uma caixa de oclusor adesivo. Porém, por ser descartável, tem que ser comprado várias vezes e pode ficar mais dispendioso ao longo do tratamento. Com base neste estudo, para que o tratamento tenha um menor custo, é sugerido o uso do oclusor plástico.

O óculos inicialmente representa grande parte do custo deste tratamento $(60,14 \%)$, mas geralmente não precisa ser trocado em curto prazo. Ao observar-se o preço dos óculos, nota-se que um deles teve um custo de R $\$ 240,00$, elevado em relação à média de preço dos óculos que foi de $\mathrm{R} \$ 118,38$. Isto se justifica por este óculos ser um bifocal "executive" utilizado em um caso de estrabismo acomodativo atípico.

A renda familiar média dos 14 pacientes avaliados foi de $\mathrm{R} \$ 560,57$ e o custo médio mensal do tratamento da ambliopia equivaleu em média a $12,20 \%$ da renda familiar mensal dos pacientes avaliados. De acordo com o censo demográfico do ano de 2000, o rendimento médio mensal dos responsáveis pelos domicílios brasileiros é de R \$769,00, variando de 488,00 a R \$ 945,00, com 12\% aproximadamente destinados à saúde e cuidados pessoais ${ }^{(17)}$.

Durante a realização do estudo constatou-se dificuldades econômicas de algumas famílias para a realização do tratamento com recurso próprio, sendo que 2 famílias obtiveram doação de oclusores adesivos e algumas famílias não tiveram gastos com transporte devido o acompanhante da criança possuir carteirinha de transporte coletivo urbano gratuito (ônibus e metrô). Notou-se que as duas famílias de menor renda per capita (família 2 e 10) obtiveram tanto transporte gratuito quanto doação de oclusor adesivo ao longo do tratamento.

Em relação ao custo do tratamento da ambliopia, este pode ser reduzido se a cura da ambliopia for alcançada em menor tempo. Para isto o ideal é o tratamento em idade precoce e bem monitorizado $^{(12)}$.

Um estudo encontrou que pacientes com idade menor de 6 anos e ambliopia leve alcançam a cura da ambliopia em menor tempo, e portanto com custo mais baixo. Os pacientes com a mesma idade e ambliopia grave obtêm a cura em maior tempo com conseqüente custo mais elevado. Desta forma, a severidade da ambliopia é um fator que pode influenciar o custo final deste tratamento ${ }^{(18)}$.

Recomenda-se triagem para ambliopia em pré-escolares por grande número de profissionais nos Estados Unidos, Canadá e Alemanha. Evidências recentes demonstraram a supe- rioridade da triagem e tratamento da ambliopia realizada antes de 37 meses ( 3 anos) de idade em comparação com a triagem realizada após 37 meses ( 3 anos) de idade. Estudo prospectivo de terapia de oclusão para ambliopia mostrou melhores resultados visuais em crianças menores de 5 anos de idade ${ }^{(19)}$.

Com o intuito de facilitar o diagnóstico e tratamento da ambliopia, a Academia Americana de Pediatria, a Associação Americana de Estrabismo e Oftalmo-pediatria e o Serviço de Prevenção de Força-Tarefa dos Estados Unidos recomendam a realização de triagem em todas as crianças entre 3 e 4 anos de idade para a detecção de ambliopia e de fatores causadores de ambliopia como estrabismo, erros refrativos e opacidades de meios $^{(20)}$. No Brasil, o Projeto Olho no Olho, realizado pelo Conselho Brasileiro de Oftalmologia em parceria com o Ministério da Educação, tem sido realizado para detectar problemas visuais nos alunos da primeira série do ensino fundamental das escolas públicas ${ }^{(21)}$. Assim, propõe-se que no Brasil se realizem projetos de triagem para detecção da ambliopia em crianças com idade entre 3 e 4 anos, quando o seu tratamento pode apresentar melhores resultados visuais e pode acarretar menores custos.

Vários especialistas na área visual têm afirmado que após a idade de 6 ou 7 anos o tratamento da ambliopia geralmente é mais difícil e mais prolongado ${ }^{(22)}$.

Considerando-se o conceito de promoção de saúde percebe-se a importância da realização de estudos de caráter econômico como este. Promoção de saúde constitui uma combinação de medidas educacionais e estruturais (ambientais e financeiras), que apresenta o objetivo primordial de incentivar estilos saudáveis de vida, mantendo o nível de saúde das populações de maneira eficaz, ética e socialmente justa ${ }^{(23)}$.

\section{CONCLUSÃO}

O tratamento de oclusão da ambliopia é eficiente e melhorou a acuidade visual do olho amblíope em 71,43\% dos casos. O custo mensal deste tratamento para o paciente é de $\mathrm{R} \$ 59,49$ e representa $12,20 \%$ da renda familiar mensal dos pacientes avaliados.

\section{AGRADECIMENTOS}

Agradeço a Dra. Célia Regina Nakanami pela escolha do assunto e ao Dr. José Augusto Alves Ottaiano pelo auxílio na elaboração do trabalho.

\section{ABSTRACT}

Purpose: To analyze the costs of therapy for amblyopia for an outpatient using occlusion of the normal sighted eye and its visual results. Methods: A prospective study was performed to evaluate the costs of therapy for amblyopia at the Strabismus Sector of the Hospital São Paulo - Universidade Federal 
de São Paulo/Escola Paulista de Medicina. Children with amblyopia ranging in age from 3 to 7 years were included in the study. The patients were followed since the diagnosis time until three consecutive visits. At the diagnosis time we prescribed glasses when necessary and total, direct and continuous occlusion of the normal sighted eye. Information about the therapy and the cost of therapy for amblyopia was collected at the following visits. To analyze the results the costs were separated into direct (glasses and occlusors) and indirect (transport, and cost of parent's/guardian's workday). Results: Fourteen patients were analyzed. The mean age was 5.21 years (from 3 to 7 years) and $7(50 \%)$ patients were male and $7(50 \%)$ female. The average of the monthly cost of amblyopia therapy was $\mathrm{R} \$ 59.49$ and the average of the annual cost was R \$ 714.47. The average monthly cost of therapy for amblyopia represented $12.20 \%$ of the monthly family income. Conclusions: The therapy for amblyopia is efficient and improves visual acuity of the amblyopic eye in $71.43 \%$ of the cases. The monthly cost of this therapy is $\mathrm{R} \$ 59.49$ being $12.20 \%$ of the patient's family income.

Keywords: Amblyopia/economics; Amblyopia/therapy; Visual acuity/physiology; Cost-benefit analysis; Sensory deprivation; Health care costs

\section{REFERÊNCIAS}

1. Mittelman D. Amblyopia. Pediatr Clin N Am. 2003;50(1):188-96.

2. Von Noorden GK, Campos EC. Binocular vision and ocular motility: theory and management of strabismus. 6th ed. St Louis: CV Mosby; 2002.

3. Diaz JP, Dias CS. Estrabismo. $4^{\text {th }}$ ed. São Paulo: Santos; 2002.

4. Pediatric Eye Disease Investigator Group. The clinical profile of moderate amblyopia in children younger than 7 years. Arch Ophthalmol. 2002;120(3):281-7.

5. Fielder AR, Irwin M, Auld R, Cocker KD, Jones HS, Moseley MJ. Compliance in amblyopia therapy: objective monitoring of occlusion. $\mathrm{Br} \mathrm{J}$ Ophtalmol. 1995;79(6):585-9.
6. Pediatric Eye Disease Investigator Group. A randomized trial of atropine vs patching for treatment of moderate amblyopia in children. Arch Ophtalmol. 2002;120(3):268-78. Comment in: Arch Ophthalmol. 2002;120(3):387-8; JAMA. 2002;287(16):2145-6

7. Keech RV, Ottar W, Zhang L. The minimum occlusion trial for the treatmen of amblyopia. Ophtalmology. 2002;109(12):2261-4.

8. National Institute of Health. The amblyopia treatment study [text on the Internet] [cited 2005 Jan 21]. Washington, march 2002. Available from: http:// www.locateadoc.com/Patient_Resources/Ophthalmology/amblyopia03.cfm

9. Better Vision for children Foundation [text on the Internet]. [cited 2004 Nov 21]. San Diego: American Association for Pediatric Ophthalmology. Available from: http://www.bvcnow.org/doctor's.html

10. Oster JG, Simon JW, Jenkins P. When is it safe to stop patching?. Br J Ophthalmol. 1990;74(12):709-11.

11. Membreno JH, Brown MM, Brown GC, Sharma S, Beauchamp GR. A costutility analysis of therapy for amblyopia. Ophthalmology. 2002;109(12): 2265-71.

12. Souza EC, Carvalho AKR, Pulchinelli A, Gass C, Rothe E, Vanzella LL, Chuffi S, et al. Cura e recorrência da ambliopia após terapia oclusiva. Arq Bras Oftalmol. 1994;57(1):16-9.

13. Tripathi A, O’Donnell NP, Holden R, Kaye L, Kaye SB. Occlusion therapy for the treatment of amblyopia: letting the parents decide. Ophthalmologica. 2002;216(6):426-9.

14. Cronemberger MF, Plut M. Ambliopia anisometrópica. Arq Bras Oftalmol. 1995;58(6):443-7.

15. Plut M, Munoz EH, Salomão SR, Consoni Filho E. Ambliopia hipermetrópica bilateral. Arq Bras Oftalmol. 1990;53(6):251-4.

16. Werner DB, Scott WE. Amblyopia case reports: bilateral hypermetropic ame tropic amblyopia. J Pediatr Ophthalmol Strabismus. 1985;22(5):203-5.

17. Instituto Brasileiro de Geografia e Estatística. Novos dados do perfil brasileiro. Apresenta textos e quadros com as características da população e dos domicílios do Brasil, segundo o Censo do IBGE 2000 [texto na Internet]. [citado $2003 \mathrm{Fev}$ 18]. Disponível em: http://www.ibge.gov.br/brasil_em_sintese/default.htm

18. Perez MF. Análise de custos no tratamento oclusivo da ambliopia por estrabismo [tese]. São Paulo: Universidade Federal de São Paulo; 2003.

19. Gandjour A, Schlichtherle S, Neugebauer A, Russmann W, Lauterbach KW. A cost-effectiveness model of screening strategies for amblyopia and risk factors and its application in a german setting. Optom Vis Sci. 2003;80(3):259-69.

20. Joish VN, Malone DC, Miller JM. A cost-benefit analysis of vision screening methods for preschoolers and school-age children. J AAPOS. 2003;7(4):283-90.

21. Conselho Brasileiro de Oftalmologia. Campanha Nacional de Reabilitação Visual. Olho no Olho [texto na Internet]. [citado 2002 Nov 10]. Ministério da Educação, CBO. Disponível em: http://www.cbo.com.br/cbo/campanhas_sociais.htm

22. Fecarotta SCS. Ambliopia: quando e como tratar. Bol Bras Ortop. 1983/1984; 11(1):25-9. Arq Bras Oftalmol. 1999;62(1):82-4.

\section{ABO ELETRÔNICO}

\section{Acesso: http://www.abonet.com.br}

